\title{
IL MODELLO SVIZZERO: UN FUTURO PER L'EUROPA?
}

\author{
di Jean Blondel
}

\section{Introduzione}

Parlar bene della Svizzera non è mai andato di moda. Se all'indomani della seconda guerra mondiale André Siegfried ne scriveva come di una democrazia modello («une démocratie témoin») (Siegfried 1947), molti altri - politici e giornalisti in particolare - l'hanno osteggiata per la sua ricchezza o per il suo neutralismo e, come sostengono alcuni, per il fatto che quella ricchezza poggiava su attività sordide legate allo stesso neutralismo. Senza arrivare a tanto, Harold Wilson, primo ministro britannico negli anni sessanta e settanta, non fu certo l'unico a stigmatizzare gli «gnomi di Zurigo»!

Come ha osservato Stein Rokkan, tuttavia, «la Svizzera si presenta come un microcosmo d'Europa: diversità linguistiche e religiose, squilibri regionali in termini di crescita economica e modelli di insediamento, autonomie strenuamente difese in un quadro di crescente e accelerata interdipendenza. Per tutte queste ragioni qualsiasi studio serio della politica elvetica rappresenta un contributo allo studio della struttura politica dell'Europa» (Rokkan 1974). Queste annotazioni sono ribadite dalle parole di un allievo di Rokkan, Daniel Seiler, che scrive: «Le istituzioni elvetiche sono il risultato di soluzioni forgiate nel fuoco di prove difficili che ebbero luogo in situazioni di crisi, ciascuna delle quali avrebbe potuto determinare la vera e propria scomparsa della Confederazione» (Seiler 1996).

$\grave{E}$ davvero sorprendente che assai poco sia stato fatto per analizzare le analogie tra ciò che la Svizzera è riuscita a realizzare nei secoli e ciò che l'Unione europea è in procinto di conseguire. Ed è singolare che l'affermazione di Seiler sia tratta da uno dei pochissimi lavori, se non il solo, che compie un accurato esame di tali analogie e trae da ciò alcune indicazioni relative 
alla linea di condotta che l'Unione europea dovrebbe adottare o eludere. Se molti svizzeri appaiono incerti rispetto all'opportunità che il loro paese aderisca all'Unione europea, ciò dipende proprio dal fatto che la Svizzera presenta numerose caratteristiche in comune con l'Unione europea. Presentando in sé le varietà e le distinzioni che l'Unione europea esibisce tra le sue varie componenti, la Svizzera avrebbe un'estrema difficoltà ad integrarsi nella più ampia unione come una qualsiasi componente tra le altre.

Questo lavoro intende avviare la discussione su ciò che Svizzera e Unione europea hanno in comune e trarre da tali comuni caratteristiche alcuni insegnamenti. A tal scopo, mi propongo anzitutto di definire quali siano i principali problemi che l'Europa si trova a dover affrontare nel suo processo unitario e di comparare tali problemi con quelli sperimentati a suo tempo dalla Svizzera. Rivolgerò quindi l'attenzione agli assetti istituzionali della Confederazione elvetica e a quelli dell'Unione europea, per capire fino a che punto essi siano simili o dissimili tra loro e, in quest'ultimo caso, se l'Unione europea avrebbe qualcosa da guadagnare imitando il modello svizzero. Infine, prenderò in considerazione alcune ulteriori caratteristiche della vita politica elvetica per valutare se l'Unione europea potrebbe trarre o no vantaggio da una loro eventuale importazione.

\section{I problemi di fondo dell'Unione europea}

Nel suo processo di più stretta integrazione, l'Europa si trova di fronte a numerose difficoltà di natura politica, sociale ed economica. I suoi problemi fondamentali hanno a che fare tuttavia con il proprio passato e con le conseguenze di tale passato. La storia europea è una storia di nazioni grandi e orgogliose di sé, separate da differenze culturali profonde, accentuate da divisioni linguistiche. Sono queste le caratteristiche che, alternativamente, impediscono agli stati-membri di giungere ad una più autentica vicinanza reciproca e di fondere la propria identità in quella dell'Unione.

Una tradizione di vecchia data. Non tutti gli stati europei sono antichi o blasonati, ma circa la metà di essi vantano una storia vecchia di parecchi secoli, durante i quali sperimentarono periodi di predominio. Per lo meno sei - Portogallo, Spagna, Paesi Bassi, Austria, Francia e Gran Bretagna - sono stati delle 
potenze mondiali; due di essi - Danimarca e Svezia - si sono caratterizzati come attori chiave in alcuni importanti passaggi del gioco politico europeo; altri due - Italia e Germania emersero nel XIX secolo occupando molto rapidamente una posizione di rilievo nel concerto delle nazioni. Ancor più che l'essere stati paesi potenti, impegnati in molti conflitti militari, ciò che appare rilevante per il processo di costruzione dell'Unione europea è tuttavia il fatto che tali paesi hanno sviluppato propri modelli amministrativi. Se alcuni erano più burocratici di altri, si trattava in ogni caso di modelli centralizzati, orientati a fornire un modo uniforme di governo all'interno di ciascun paese, e ciò ha determinato profonde differenze amministrative tra i diversi paesi.

Queste caratteristiche hanno contribuito a mantenere vivo il ricordo del ruolo esercitato nel passato. Se il declino della maggior parte dei paesi europei e la sconfitta di Italia e Germania alla fine della seconda guerra mondiale hanno ridimensionato la loro «arroganza» nelle relazioni reciproche e nei rapporti col resto del mondo, l'orgoglio per i propri risultati amministrativi è rimasto tale. Gran Bretagna e Francia, inoltre, hanno continuato a credere in un proprio ruolo su scala mondiale. In modi diversi, questi due paesi hanno così mantenuto le distanze nei confronti del «corsetto» che l'Unione europea potrebbe imporre loro, inducendo gli altri stati a comportarsi in modo analogo. L'Europa continua ad essere considerata dai più come un'«Europa di nazioni», le quali non intendono separarsi dalla propria anteriore centralità storico-politica e, in molti casi, si sentono orgogliose dei risultati amministrativi conseguiti all'interno dei propri confini nazionali.

Diversità culturali. I singoli stati europei confidano sul proprio status tanto più in quanto la maggior parte di essi può pretendere di incarnare una tradizione culturale, che, pur non mancando di aspetti controversi, rappresenta per la massa dei cittadini come per le élites un punto di riferimento concreto. A livello «alto», tale cultura è correlata alla letteratura, alle arti, alla musica, e quindi allo sviluppo del pensiero e viene percepita come un patrimonio da salvaguardare, il che confuta l'idea stessa dell'esistenza di un'unica comunità politica europea. A livello di massa queste diversità vertono sulla centralizzazione o il decentramento delle attività, su variazioni relative al tema città/ campagna, e soprattutto su differenze religiose. Una delle dimensioni di questo spartiacque «culturale» separa il Nord pro- 
testante - considerato da chi vi appartiene più onesto, più propenso a lavorare duramente, più democratico e più avanzato sul piano sociale ed economico - dal Sud cattolico. Tutto ciò lascia inalterate alcune tensioni di fondo, non senza conseguenze tangibili: in due referendum diversi, i norvegesi hanno votato contro l'ingresso del loro paese nell'Unione europea; sempre per via referendaria, i danesi si sono espressi inizialmente contro il Trattato di Maastricht, anche con motivazioni relative alle maggiori diseguaglianze subite dalle donne nei paesi dell'Europa del Sud.

Differenze linguistiche. Le diversità culturali si nutrono, evidentemente, di differenze linguistiche. Il processo di integrazione europea iniziò con quattro lingue per sei soci fondatori; quando i membri della Comunità diventarono nove, le lingue crebbero a sei; passarono a nove con dodici partner e poi a undici quando i paesi sono diventati quindici. Questa sorta di babele è aggravata dai sottintesi culturali che alcuni, in particolare i francesi, riconducono alla lingua. Mentre per la larga maggioranza degli europei l'inglese è diventata la lingua franca, il governo di Parigi ha infatti mantenuto un'ostinata politica di promozione della lingua francese. Tale politica ebbe la meglio fino all'ingresso della Gran Bretagna nella Comunità, diventando sempre meno giustificabile negli anni settanta. Poiché la lingua, e il francese in particolare, è il veicolo di un'intera cultura, rappresenta un importante fattore di divisione tra i membri dell'Unione. Il punto di vista francese sull'importanza culturale della lingua, inoltre, ha teso ad estendersi ai rappresentanti degli altri stati-membri; e alla questione linguistica si trova strettamente associata la lotta, che magari lo stato sostiene con il suo peso e la sua autorità, per il mantenimento di un cinema nazionale e perfino di una filosofia nazionale contro il cinema e la filosofia di altre nazioni.

L'influenza di queste differenze sulla struttura politica dell'Unione europea. Le divisioni cui si è fatto fin qui riferimento hanno avuto e continuano ad avere profonde conseguenze politiche sul processo di integrazione. Quattro di tali conseguenze meritano di essere ricordate: gli infiniti dibattiti sul «federalismo»; il persistente carattere nazionale, anziché comunitario, delle istituzioni sociali e politiche chiave; il fatto che le strutture dell'Unione europea restino deboli o mantengano un carattere di fatto nazionale; la nozione di un «deficit democratico» insormontabile esistente all'interno dell'Unione europea. 
L'opposizione (a volte) stridula alla «federalizzazione» dell'Europa scaturisce direttamente dal senso di identità culturale avvertito in molte nazioni, nonché dalla persistente importanza dello stato. Coloro che con maggior forza avversano l'idea di «federalizzare» l'Europa tendono spesso ad assumere tale posizione perché presumono la superiorità culturale e/o amministrativa della propria nazione. Non sorprende, pertanto, che tale avversione si presenti con maggiore forza nel Nord Europa, dove si respira un diffuso senso di superiorità culturale e sociale nei confronti degli altri europei. Sul punto la Francia è ambivalente: da un lato, vi è un forte orientamento a salvaguardare l'identità del paese; dall'altro, non manca l'ambizione, altrettanto forte, di convincere gli altri europei della bontà delle proprie idee e quindi di indurli a seguire la guida francese!

Lo scontro sulla federalizzazione investe soprattutto aspetti simbolici, poiché non molti sembrano aver compreso quanto il concetto di federalismo sia vago e quindi oscuro. In effetti, le accezioni di federalismo sono tante quanti sono i paesi federali; i federalisti «veri» considerano infatti la maggior parte dei paesi federali tali soltanto di nome, non solo nella realtà politica, ma anche nelle clausole costituzionali. A proposito dei paesi dell'America latina e perfino dell'India, Wheare parlò negli anni sessanta di «quasi-federalismo» (1966). Si potrebbe così sostenere con qualche fondamento che il carattere sovranazionale di alcune istituzioni e prassi dell'Unione europea costituisca già una forma embrionale di federalismo.

D'altro canto, persino paesi considerati senza ombra di dubbio federali - Stati Uniti, Australia o Canada - hanno sempre adottato o sono giunti ad adottare prassi «intergovernative» fra le proprie unità componenti (stati o regioni) con riferimento a una congerie di materie che spaziano dall' istruzione all'economia. Ciò suggerisce che l'intergovernatività fa parte a pieno titolo della realtà del federalismo: la questione cruciale non è se le decisioni sono prese da un organo federale, ma se siano prese in comune. Il dibattito relativo al grado in cui l'Unione europea potrebbe $o$ non potrebbe diventare una federazione può quindi essere considerato privo di contenuto. Costituisce tuttavia una fonte di divisioni profonde, soprattutto per la connotazione emozionale del termine e per gli effetti che il federalismo si ritiene possa avere sull'autonomia dello stato e sulla salvaguardia delle culture nazionali.

In concreto, la questione del riconoscimento dell'Unione 
europea come attore rilevante può essere posta in relazione con la sede in cui, nei gruppi e nei partiti che operano a livello europeo, il processo decisionale ha luogo. In quasi tutti i casi, la sede è una sede nazionale, mentre il livello europeo rappresenta di fatto soltanto il luogo in cui i responsabili nazionali convergono quando una specifica decisione relativa a una questione di interesse europeo dev'essere assunta. Ciò avviene sia per le «confederazioni» imprenditoriali sia per quelle sindacali, le quali soffrono le barriere linguistiche, tendono a interessarsi più delle attività d'impresa che delle questioni sociali e sono così ancor più deboli delle prime. Forse per ragioni analoghe, anche le associazioni volontarie sono meno interessate a sedi europee che non a sedi nazionali, o, in certi casi (Greenpeace; Amnesty International), mondiali. Ciò è vero anche per i partiti, che a livello europeo restano assai deboli. I gruppi partitici del Parlamento europeo sono spesso aggregazioni di comodo e anche quando non è così - come per il Partito socialista europeo, per il Partito popolare europeo e, in minor misura, per il Partito liberale europeo - si tratta di «confederazioni» presenti al di fuori dell'arena parlamentare soltanto nelle settimane che precedono le elezioni europee e soltanto per adottare programmi invero piuttosto generici. I partiti europei non sono partiti in senso proprio: il reclutamento dei candidati avviene a livello nazionale e le campagne elettorali, anche quella per le elezioni europee, si sviluppano a livello nazionale. Gli attori che dovrebbero modellare la vita politica europea, insomma, sono per l'essenziale diretti su base nazionale.

Dal canto loro, come si è detto, le strutture dell'Unione europea tendono ad essere deboli oppure poco europee nel loro carattere. Delle quattro principali istituzioni - il Consiglio, la Commissione, il Parlamento, la Corte di giustizia - la Corte è forse non solo quella più solida, ma anche quella più incline a «pensare» su scala europea. In effetti, per certi rispetti, ha indebolito la «sovranità» degli stati-membri. La Commissione è stata invece in parte indebolita dal Consiglio, rappresentante par excellence degli stati-membri. La scarsa implicazione della Commissione in due dei tre capisaldi dell'Unione (la politica di sicurezza; gli affari esteri) è indicativa della forza dispiegata dai governi nazionali nel mantenere la loro presa sul decision-making dell'Unione europea. Neanche il Parlamento europeo, infine, è in grado di fornire una prospettiva autenticamente europea nei confronti dei governi degli stati-membri. Ciò è dovuto non solo 
alla sua intrinseca debolezza, peraltro minore che in passato, ma anche al fatto che i suoi membri, come si è detto, sono selezionati da partiti nazionali e sono spesso impazienti di far ritorno alla politica nazionale. Le istituzioni dell'Unione europea allo stesso modo dei partiti, dei gruppi di interesse e delle associazioni volontarie - non sono in definitiva che appendici delle istituzioni nazionali.

La diffusa diffidenza nei confronti delle tendenze «federaliste», il limitato grado in cui gruppi e partiti possono dirsi «europei», la scarsa connotazione «europea» di istituzioni formalmente tali si traducono in una opinione altrettanto diffusa sul basso grado di legittimità dell'Unione europea. Se un'aperta opposizione all'Unione, salvo che in qualche stato-membro, è assai contenuta, si deve riconoscere che il sostegno è un sostegno piuttosto passivo. Questa passività è apparsa a lungo evidente nei dati Eurobarometro: l'incidenza degli intervistati che proclamava la propria indifferenza di fronte all'ipotesi di dissoluzione dell'Unione non era inferiore a quella di coloro che, di fronte a tale evenienza, si sarebbero dichiarati «dispiaciuti» 1 . L'Unione europea sembra essere dunque più tollerata che non autenticamente apprezzata.

Questo stato di cose è stato spesso ricondotto al «deficit democratico» di cui, come spesso si afferma, l'Unione europea sembra soffrire. Tale deficit, evidentemente, era consistente finché il Parlamento non era eletto direttamente; continua ad essere cospicuo con un Parlamento dai poteri limitati, anche se più sostanziali, a fronte di un Consiglio che definisce i propri compromessi a porte chiuse e di una Commissione considerata tecnocratica e produttrice di direttive la cui utilità appare spesso discutibile. Che il «deficit» esista è indubbio; ciò di cui è lecito dubitare è il fatto che l'opinione pubblica veda le cose in questi termini. I dati di sondaggio non hanno mai suggerito che la scarsa democraticità delle istituzioni dell'Unione europea catalizzasse l'attenzione dell'elettorato europeo'.

$1 \mathrm{Nel}$ 1994, ad esempio, tra quelli che erano allora i dodici stati-membri dell'Unione europea, il $37 \%$ degli intervistati affermava che il dissolvimento dell'Unione l'avrebbe lasciato indifferente, il $45 \%$ sosteneva che ne sarebbe rimasto dispiaciuto, l'11\% che ne avrebbe tratto sollievo. L'8\% dichiarava di non saper rispondere. Cfr. Eurobarometro 41.1 .

$2 \mathrm{Nel} 1994$, ad esempio, il livello di soddisfazione per il grado di democrazia esistente nell'Unione europea era appena inferiore al livello di soddisfazione per il grado di democrazia esistente nel paese dell'intervistato: $42 \%$ degli intervistati nel primo caso, $48 \%$ nel secondo e identici livelli di soddisfazione nel $50 \%$ delle risposte. 
Il disinteresse nei confronti delle questioni europee è, tuttavia, un'evidenza inoppugnabile. Alle elezioni europee si partecipa assai meno che alle elezioni nazionali. Alcuni autori hanno proposto di considerare le europee come elezioni di «secondo ordine», in cui gli elettori votano più per punire o mettere sull'avviso i governi nazionali che non per specifiche questioni di carattere europeo (Reif 1985). Tali questioni, peraltro, risultano spesso vaghe e il comportamento dei partiti al Parlamento europeo non è tale da ricondurle a distinzioni nette, immediatamente intelligibili. La limitata salienza dell'Europa rispetto agli statimembri riporta così l'attenzione al problema chiave dell'Unione: prima di poter guadagnare una propria autentica identità, il suo «peso» nella testa dei cittadini dovrà assumere ben altra consistenza.

\section{Svizzera e Unione europea: le sfide comuni}

Fin qui, dunque, i difficili ostacoli che l'Unione europea deve superare. Di simili svantaggi anche la Svizzera ha sofferto $\mathrm{e}$ in parte continua a soffrire. In varie zone del paese si sono spesso manifestati problemi di identità nazionale; $i$ cantoni hanno continuato a nutrire sentimenti di diversità altrettanto acuti di quelli esistenti nel contesto europeo e superarne le conseguenze non è stato facile e ha richiesto tempo, come è prevedibile che accada nel caso dell'Unione europea.

I ventisei cantoni che compongono la Svizzera sono veri e propri stati. Il processo di integrazione che ebbe inizio con il patto confederale nel 1291 non ha cancellato le sensibilità locali. In effetti tra il 1291 e la rivoluzione francese accadde ben poco che potesse ridurre l'indipendenza cantonale: la Confederazione crebbe soltanto in dimensioni e numero di associati. Nel 1789 gli allora 13 cantoni costituivano un'alleanza difensiva, più simile alla Nato o all'Unione Euro-occidentale che non alla stessa Unione europea. Il quadro non cambiò dopo la caduta dell'Impero francese, nonostante l'insediamento di uno stato svizzero da parte di Napoleone. Il Congresso di Vienna ristabilì la vecchia Confederazione, che comprendeva ormai 22 cantoni, tre dei quali del tutto nuovi (tra cui Ginevra, che perse la sua indipendenza) e sei frutto di secessioni di cantoni già esistenti (in particolare Berna). I cantoni mantennero la loro «totale indipendenza» fino al 1848. A rappresentare $\mathrm{i}$ cantoni vi era 
soltanto una Tagsatzung che decideva all'unanimità. La costituzione del 1848, tuttavia, prevedeva un esecutivo e un corpo legislativo federali e conferiva a tali organi un certo numero di importanti, pur se limitati, poteri. Peraltro, come ha osservato Linder, «da parte dei padri della costituzione federale [del 1848] vi era piena convinzione che i cantoni non avevano perso la loro sovranità, ma soltanto parte delle proprie prerogative [...] Determinati a conservare un ampio grado di autonomia politica, i cantoni mantennero molti dei loro poteri e la partecipazione al processo decisionale della nuova federazione» (1994, 39). Come spesso nel caso dell'Unione europea, la questione restò irrisolta e la Svizzera, particolare interessante, mantenne nella sua denominazione il termine «confederazione». Buona parte degli attuali 26 cantoni vantano un passato nobile e antico, dal cantone di Uri, le cui origini risalgono all'alleanza contro la «tirannide» austriaca, alla «repubblica e cantone» di Ginevra, dove la fede calvinista si affermò e da cui si diffuse in larga parte d'Europa.

I cantoni rimasero non solo arbitri delle proprie questioni interne, ma continuarono a presentare sul piano amministrativo diversità cui non intendevano rinunciare. In alcuni, il decentramento era considerevole e ai comuni erano riconosciuti ampi poteri decisionali; in altri, ad esempio Berna e Vaud, il governo cantonale esercitava invece il ruolo principale (Allemann 1985). In Svizzera l'approccio al concetto di stato è dunque a geometria variabile, con un'evidente analogia con la varietà di approcci al concetto di stato che sussiste nell'Unione europea.

Come nell'Unione europea, inoltre, le diversità culturali sono evidenti e sono avvertite come tali. In passato la contrapposizione tra cattolici e protestanti aveva costituito il principale conflitto politico, culminato nella guerra civile del 1847 - la guerra del Sonderbund - scaturita dal tentativo di secessione dei cattolici, che avevano ritenuto posti a repentaglio i propri diritti. La secessione fallì, ma i vincitori riconobbero che la Svizzera avrebbe potuto continuare ad esistere soltanto se le differenze e non solo quelle religiose - fossero state pienamente riconosciute. Da questa propensione a convivere con divisioni di ogni tipo emerse il «consociativismo», una delle caratteristiche chiave della moderna politica svizzera. Ciò non toglie che le divisioni culturali continuino a sussistere.

Intanto, come aveva osservato Rokkan, tra i diversi cantoni non mancavano, e persistono tuttora, sensibili differenze nei va- 
lori e nello stile di vita, correlate in molti casi a differenze socio-economiche. Lo scarto si presenta assai sensibile tra il «cuore» della Confederazione - $\mathrm{i}$ tre cantoni che diedero inizio al processo di integrazione - e le grandi città aperte al mondo esterno (Zurigo, Basilea e Ginevra; non Berna, che resta più tradizionale e inward-looking). Ne costituisce un riscontro puntuale il diverso orientamento elettorale, che si manifesta in particolare in quei referendum nazionali che investono le relazioni tra Svizzera e resto del mondo (Eschet-Schwarz 1989).

Le differenze linguistiche sostengono queste diversità culturali. Si tratta di differenze che vanno al di là delle tre principali lingue a cui di solito ci si riferisce. Se il tedesco è infatti parlato ufficialmente da più del $70 \%$ della popolazione, il francese da circa il $20 \%$ e l'italiano dal $7 \%$, vi sono anche due lingue romanze parlate in alcune vallate del cantone dei Grigioni e un certo numero di «dialetti» tedeschi, poiché lo Schwitzertütsch, diffuso anche come lingua scritta, presenta numerose varietà. La lingua è quindi un elemento chiave dell'identità cantonale. Ciò è vero soprattutto perché queste lingue sono geograficamente delimitate. Come nel caso dell'Unione europea, molti stati (vale a dire i cantoni) sono monolingui; in tre di essi (Berna, Friburgo, Vallese) si parlano due lingue (francese e tedesco) - anche se soltanto le città sono effettivamente bilingui; nel cantone dei Grigioni si parlano tre lingue (tedesco, italiano e ladino), ma anche in questo caso con limitate aree trilingui. Ed è interessante notare che il cantone del Giura, l'ultimo nato, creato negli anni settanta, è stato il frutto della secessione della maggior parte dell'area francofona del cantone di Berna, le cui autorità erano state accusate di imperialismo linguistico e culturale.

In termini di «costruzione dello stato», di diversità culturali, di differenze linguistiche, tra Svizzera e Unione europea vi sono, insomma, considerevoli affinità. Se in Svizzera alcuni di questi aspetti, ad esempio la varietà delle strutture statuali, sono meno netti che in passato, altri rimangono pronunciati malgrado i cittadini svizzeri convivano da molti decenni, il che sembra confermare la citata affermazione di Rokkan secondo cui la Svizzera sarebbe un «microcosmo» d'Europa.

Si potrebbe tuttavia osservare che tra caso elvetico e Unione europea le dissimiglianze non sono poche. Vi è, in primo luogo, una differenza di scala che potrebbe considerarsi talmente importante da inficiare qualsiasi comparazione tra le due entità. Il fatto che la Svizzera abbia dimensioni territoriali e demografi- 
che assai più ridotte dell'Unione europea non implica però che in Svizzera la consapevolezza delle differenze sia meno marcata, né, soprattutto, che in passato sia stata meno marcata di quanto non lo sia oggi nell'Unione europea. Nella prima metà del XIX secolo i cittadini che si stabilivano al di fuori del proprio cantone di nascita erano assai pochi e la mobilità era disincentivata da regole che facevano della cittadinanza una prerogativa esclusivamente municipale e cantonale. Come l'Unione europea negli anni settanta, al fine di rompere le barriere esistenti fino a quel momento, la costituzione svizzera del 1848 sancì la libertà di movimento delle persone.

Tra i cantoni vi sono inoltre considerevoli differenze di carattere demografico. Si tratta, in proporzione, di differenze non meno cospicue di quelle esistenti tra gli stati-membri dell'Unione europea, eccezion fatta per il Lussemburgo: per i cittadini di cantoni con poche migliaia di valligiani come Schwyz e Appenzello, Zurigo, con più di un milione di abitanti, è davvero una «grande potenza». Nella Confederazione, il peso di Zurigo, Berna o Vaud è quindi assai più grande di quello dei piccoli cantoni, aspetto questo che suscita di per sé sentimenti di diversità e reazioni difensive, così come accade nell'Unione europea.

Una seconda, importante differenza tra Svizzera e Unione europea potrebbe essere colta nel fatto che il dispositivo originario della Confederazione è stato la politica di difesa, dapprima nei confronti dell'Austria e più tardi contro le grandi potenze europee che circondavano il paese. Per questo motivo, lo sviluppo della Confederazione potrebbe essere assimilato a quello della Nato, che ha legato i paesi europei in funzione difensiva, piuttosto che a quello dell'Unione europea.

Anche nel caso della costruzione dell'Unione europea, tuttavia, le considerazioni relative alla difesa sono state tutt'altro che assenti. Se i mezzi utilizzati sono stati principalmente economici, lo scopo principale dell'unificazione europea, soprattutto nelle fasi iniziali, è stato di sicurezza, sia all'interno che verso l'esterno. La Comunità economica del carbone e dell'acciaio e i successivi trattati che hanno legato gli stati europei hanno avuto lo scopo implicito di prevenire conflitti armati tra tali stati e di porre i paesi dell'Europa occidentale nelle condizioni di rispondere alla sfida del comunismo sovietico. Se non fosse esistita una cortina di ferro non è detto che l'Unione europea sarebbe sorta egualmente, né che si sarebbe sviluppata con la rapidità con cui si è sviluppata. 
$\mathrm{Su}$ questo stesso terreno, tra Svizzera e Unione europea si impone un ulteriore parallelo. In apparenza tra la neutralità della Svizzera e l'attivismo internazionale di alcuni stati-membri dell'Unione europea, Gran Bretagna e Francia in particolare, sembra esserci una differenza significativa. In realtà, l'incapacità di andare al di là di risoluzioni piuttosto banali a causa delle divisioni tra i singoli stati-membri rende l'Unione europea spesso impotente su molte questioni di politica estera. Ciò ricorda da vicino le condizioni della Svizzera, i cui cittadini hanno puntato sul neutralismo come unica possibilità di superare i contrasti tra cantoni le cui simpatie andavano in direzioni diverse.

Tutto ciò mostra come il parallelo tra Svizzera e Unione europea non manchi di fondamento. Un ampio numero di caratteristiche comuni controbilancia le dissimiglianze che potrebbero sembrare sussistere tra le due entità. La Svizzera, inoltre, è l'altro unico caso disponibile di un processo di unificazione che prende avvio con un accordo tra unità componenti che hanno relativamente poco in comune. È per questo motivo che il federalismo «confederale» elvetico si trovò a dover inventare strutture diverse da quelle di altre federazioni, compresi gli Stati Uniti e nonostante gli estensori della costituzione del 1848 avessero assunto la costituzione americana a modello di riferimento.

A volte si tende a considerare il federalismo uno strumento per passare da un assetto confederale a uno stato unitario. In realtà avviene proprio il contrario: le federazioni tendono ad essere soluzioni imposte dall'alto per prevenire la disgregazione di uno stato e per conciliare differenze considerate insanabili tra parti di ciò che fino a quel momento era uno stato unitario. Il federalismo, infatti, è stato imposto da un potere coloniale in via di ritirata per mantenere l'unità di una nazione di nuova indipendenza (è il caso di Canada, Australia, Nigeria, Malaysia); è stato introdotto, in contesti autoritari, per sedare spinte secessioniste (Unione Sovietica, Jugoslavia, Cecoslovacchia); è stato introdotto, attraverso un accordo, perché un sistema unitario non sembrava più (Brasile, Messico, Argentina, Venezuela), o non era più (Belgio) in grado di funzionare, o non appariva in grado di garantire la continuità di un sistema liberale (Austria e Germania post-1945: un federalismo imposto dall'alto in entrambi i paesi). Gli Stati Uniti sono stati il solo paese, oltre la Svizzera, in cui l'accordo federale ha rappresentato il risultato di un autentico accordo tra le unità componenti. Le tredici co- 
lonie che diedero vita agli Stati Uniti erano però affini tra loro non solo linguisticamente, ma anche per impianto politico-amministrativo. Gli Stati Uniti mutuarono infatti i propri principi di fondo dalla Gran Bretagna, sulla base di essi combatterono la Gran Bretagna e tali principi diventarono la base ideologica dell'identità americana.

La storia e la struttura sociale della Svizzera sono completamente diverse. Prima che l'adozione della costituzione nel 1848 introducesse legami più stretti e istituzionalizzati tra i cantoni, trascorse un periodo di tempo assai lungo, durante il quale $\mathrm{i}$ cantoni si avvicinarono tra loro e parti di cantoni si divisero per formare nuovi cantoni. Negli Stati Uniti, al contrario, l'aggregazione di nuovi stati si realizzò dopo che i tredici soci fondatori ebbero adottato la costituzione, cosicché i nuovi stati non poterono far altro che adottare il modello istituzionale dei primi. La Svizzera è quindi il solo esempio di quello che il federalismo sembrerebbe dover essere ma in realtà non è, cioè uno strumento per tenere assieme unità significativamente diverse attraverso «accordi amichevoli» (Steiner 1974). Ciò rende la comparazione tra Svizzera e Unione europea non soltanto fondata, ma addirittura necessaria.

\section{Le caratteristiche del sistema politico elvetico}

In effetti, le difficoltà identificate in relazione al caso dell'Unione europea - ovvero i problemi posti dall'idea federale, la maggior vitalità delle istituzioni politiche di livello nazionale rispetto a quelle dell'Unione europea, il basso grado di legittimità dell'Unione europea rispetto ai propri stati-membri - sono state tutte sperimentate dalla Svizzera, e in parte continuano ad esserlo. L'idea che la Svizzera sarebbe diventata una federazione, ad esempio, richiese molto tempo per radicarsi. Se si assumono come termine di riferimento le definizioni dei manuali, quello elvetico non sembra essere un «vero» federalismo. In Svizzera, il federalismo è cresciuto per gradi. Alla fine del XIX secolo, la federazione erogava pochi servizi; le due guerre mondiali, pur non toccando direttamente il paese, determinarono un'estensione dei poteri del governo federale rispetto ai governi dei singoli cantoni, che tuttavia continuano ad essere per più rispetti gli attori decisionali più influenti (Knapp 1987). Il federalismo elvetico, inoltre, non si basa, come quello americano, su una ben 
definita divisione tra poteri statali e poteri federali, ma presenta un'ampia zona grigia in cui le funzioni dei due livelli si sovrappongono, e quindi un intricato insieme di relazioni tra i diversi livelli. Per questi motivi si avvicina più al federalismo tedesco che a quello americano.

In effetti, i cantoni continuano a rappresentare l'arena in cui la vita politica trova principalmente svolgimento e rivestono perciò un ruolo chiave. Rispetto all'Unione europea, i gruppi di interesse presentano in Svizzera una maggiore coesione. Tanto le associazioni imprenditoriali che i sindacati contano infatti su attive organizzazioni nazionali, malgrado le diversità connesse al profilo economico differenziato dei singoli cantoni. Su questo terreno, la differenza più significativa tra Unione europea e Svizzera è legata alle caratteristiche delle organizzazioni sindacali, anche perché in Svizzera un'attività sindacale significativa si manifestò molto tempo dopo l'adozione della costituzione del 1848 e richiese una fase di sviluppo e di graduale unificazione. $\mathrm{Al}$ tempo del primo conflitto mondiale, l'unità sindacale era un fatto compiuto e il ruolo dei sindacati culminò nella Convenzione per la concordia del lavoro del 1937, una politica senza eguali nel mondo liberale, che prevedeva la rinuncia allo sciopero e alla serrata come mezzi di pressione nei conflitti di lavoro (Linder 1994, 30-31).

I cantoni continuano ad essere più importanti anche a livello partitico. Con la parziale eccezione del Partito socialista, i partiti svizzeri hanno conservato una caratterizzazione cantonale e si presentano a livello nazionale come matrimoni di convenienza. Sul piano organizzativo, radicali, cristiani e liberali - i tre partiti che assieme ai socialisti dominano la vita politica elvetica - sono strutturati a livello cantonale. A livello cantonale avviene la selezione dei candidati, sia per le elezioni cantonali che per quelle nazionali; sono decise le politiche, cosicché uno stesso partito - è in particolare il caso del partito cristiano può trovarsi collocato a destra in un cantone e al centro-sinistra in un cantone diverso; e vengono tradizionalmente raccolti anche i finanziamenti partitici.

Pertanto, il governo di ciascun cantone è un governo sui generis. I cantoni più piccoli hanno adottato forme di democrazia diretta, e i loro cittadini si radunano una volta l'anno per approvare le leggi e il bilancio. I cantoni più grandi presentano invece una struttura istituzionale a più livelli, che spesso sovrintende all'attività dei comuni (Allemann 1985). I leader cantonali 
possono quindi considerarsi a buon diritto almeno altrettanto influenti dei loro colleghi di livello nazionale.

A livello della Confederazione, in effetti, la distribuzione del potere dipende da quanto avviene a livello cantonale assai più di quanto di solito non avvenga in altre federazioni. Ciò si spiega non solo perché la Camera alta - il Consiglio di Stato - rappresenta i cantoni ed è formata, sul modello del Senato americano, da due membri per cantone, ma anche perché i membri della Camera bassa - il Consiglio nazionale - restano per lo più fedeli al proprio partito cantonale. Il ruolo dei cantoni, o almeno dei principali di essi, si fa sentire anche nel Consiglio federale, l'esecutivo elvetico, poiché i sette membri che lo compongono sono nominati seguendo una formula «magica» che consente ai cantoni più grandi di decidere, in base a un principio di rotazione, chi sarà eletto se, e quando, alla fine del proprio mandato, un membro dovesse ritirarsi. La composizione del Consiglio federale, eletto ogni quattro anni dalle due camere sulla base di un accordo predefinito, non soltanto tiene conto di una ripartizione 2-2-2-1 tra i quattro maggiori partiti e (grosso modo) 4-21 tra le tre principali comunità linguistiche, ma si fonda anche su un criterio di rappresentanza dei principali cantoni all'interno dell'esecutivo.

Il Consiglio federale è un organo pienamente collegiale, il cui Presidente (il Presidente della Confederazione) ruota annualmente. Le decisioni sono assunte sulla base di accordi tra $\mathrm{i}$ membri, i quali riflettono indirettamente il punto di vista dei partiti, delle comunità linguistiche e dei cantoni. Il Consiglio federale, inoltre, può soltanto formulare proposte al Parlamento, che, come si è detto, dipende dal potere cantonale ed è vincolato inoltre dalle procedure referendarie, ulteriori strumenti attraverso cui vari gruppi - e in particolare gruppi di livello cantonale - possono limitare il potere delle autorità confederali. Le politiche, in tal modo, potranno essere adottate soltanto se si saranno dimostrate condivise da una larga maggioranza dei principali segmenti di popolazione. Non sorprende, quindi, che il Consiglio e il Parlamento federali tendano di fatto a ratificare proposte preparate da numerose commissioni nelle quali i diversi interessi coinvolti sono rappresentati. Questo assetto, estremamente consociativo, rende assai limitato il margine di manovra delle autorità della Confederazione. Rispetto alla struttura istituzionale dell'Unione europea vi è un parallelismo evidente, con due differenze principali: nell'Unione europea tutti 
gli stati-membri - e non solo i più grandi di essi secondo un criterio di rotazione - detengono un seggio nella Commissione europea; il Presidente della Commissione europea rimane in carica per un intero mandato di cinque anni.

Il carattere consociativo del sistema elvetico dà luogo a forme di intragovernatività e a forme di stretta associazione tra governo federale e governi cantonali. $\mathrm{Da}$ tale nesso emerge un grado di «sussidiarietà» non troppo diverso da quello che l'Unione europea promuove o è costretta ad adottare. In Svizzera, più specificamente, distinguere ciò che è strictu sensu «sovranazionale» da ciò che invece è «intergovernativo» è spesso difficile. Ad eccezione delle poste e delle ferrovie, in effetti, l'amministrazione federale si riduce a poca cosa: le attività dell'amministrazione pubblica sono infatti condotte per lo più da corpi cantonali. Se si considera che nell'ultimo secolo e mezzo la Confederazione ha gradualmente acquisito maggiori prerogative, ciò che sorprende non è tanto che la Svizzera sia più «federale» dell'Unione europea, ma che i cantoni abbiano conservato così tanti poteri. Tale stato di cose può essere attribuito soltanto al fatto che tutti i gruppi di una qualche rilevanza hanno visto preservati $i$ loro interessi e che, tra tali gruppi, $i$ cantoni sono stati tra i più influenti, riconosciuti dai più come arene privilegiate. Il fatto non è tanto che la maggior parte dei servizi pubblici sono erogati dai cantoni anziché dal governo federale - una situazione simile a quella tedesca e differente da quanto avviene negli Stati Uniti, dove ci sono amministrazioni parallele degli stati e del governo federale. Ciò che rende l'amministrazione cantonale più influente $\mathrm{e}$ indipendente dalle entità omologhe di altre federazioni è il fatto che alcuni dei servizi in questione sono, o sono stati a lungo, amministrati nei diversi canto$\mathrm{ni}$ in modi diversi. Non è soltanto il caso dell'istruzione pubbli$\mathrm{ca}$, che, come in altre federazioni, è rimasta, anche a livello di istruzione superiore, una questione di esclusiva competenza cantonale; è anche il caso degli schemi pensionistici nazionali istituiti nel 1948, che, nel quadro di alcuni comuni principi generali, hanno consentito ai cantoni di operare liberamnte.

Le strutture politiche della Svizzera, insomma, dipendono in modo significativo dai cantoni. Questo tratto è rimasto finora inalterato perché l'identità elvetica, ancora una volta allo stesso modo di quanto avviene nel caso dell'Unione europea, si è sviluppata con lentezza, essendosi affermata dopo, e in larga parte attraverso, l'identità cantonale. Un esempio cruciale di questo 
sviluppo è costituito dalle norme che regolano la cittadinanza. Acquisire la cittadinanza svizzera in quanto tale non è possibile. A tal fine è infatti previsto un processo che inizia a livello municipale e prosegue a livello cantonale; soltanto dopo che il municipio e il cantone hanno «accettato» la possibilità che uno straniero possa diventare cittadino, quest'ultimo può adire il livello confederale. La cittadinanza svizzera, condizionata com'è al gradimento cantonale (e municipale), si caratterizza così come una cittadinanza effettivamente «duale». Tale sviluppo presenta molte analogie con le proposte elaborate in relazione all'istituzione di un'ipotetica cittadinanza europea, che dovrebbe dipendere ed essere condizionata dal possesso (o dall'ottenimento) della cittadinanza di uno degli stati-membri.

La Confederazione svizzera presenta, rispetto all'Unione europea, un più solido grado di legittimità. Considerando che la costituzione elevetica è stata adottata ormai già un secolo e mezzo fa, la cosa non può sorprendere. È interessante osservare, tuttavia, che il livello di partecipazione alle elezioni federali è piuttosto modesto, allo stesso modo di quanto avviene alle elezioni per il Parlamento europeo (Aubert 1979; Sidjanski 1979). Per dar conto del basso turnout, talvolta si è chiamata in causa la frequenza con cui i cittadini svizzeri sono chiamati al voto, soprattutto a causa dei numerosi referendum, cantonali e confederali, ai quali si chiede loro di pronunciarsi. Occorre tuttavia notare - e ciò dà luogo a un ulteriore parallelo con l'Unione europea - che mentre dalle elezioni cantonali (e municipali) può scaturire un cambiamento della maggioranza di governo, ciò non avviene a livello federale, poiché la formula «magica» stipula che il Consiglio federale sia composto sempre e comunque da rappresentanti dei quattro principali partiti. A livello federale la posta in gioco appare quindi piuttosto bassa. Identica conclusione vale per le elezioni del Parlamento europeo: essendo il risultato, in un certo senso, conosciuto in anticipo, gli incentivi al voto sono assai modesti. Nella misura in cui ciò che deve prevalere è la soddisfazione di tutti i principali gruppi, tale esito è inevitabile. Ciò non di meno, ne scaturiscono conseguenze nei rapporti tra i cittadini svizzeri e i vertici del governo elvetico, così come nei rapporti tra cittadini europei e Unione europea. 
Cosa la Svizzera potrebbe insegnare

I paralleli tra Svizzera e Unione europea sono dunque palesi. Poiché la Svizzera ha prosperato pur soffrendo dello stesso tipo di svantaggi che affliggono l'Unione europea, potremmo fare un passo ulteriore e chiederci quali lezioni la Svizzera può fornire perché l'Unione europea possa anch'essa prosperare. Tali lezioni possono essere riferite a quattro aree distinte: gli assetti decisionali, la politica linguistica, la politica estera, i referendum.

Mantenere una struttura decisionale non ben definita. L'Unione europea ha adottato istintivamente un insieme di assetti decisionali ed anche alcune prassi assai simili a quelli che caratterizzano l'esperienza elvetica. Come in Svizzera, i processi decisionali fondati sulla ricerca di un ampio consenso sono infatti prevalenti, come se fosse da tutti riconosciuto che l'Unione non avrebbe potuto svilupparsi, ed anzi avrebbe corso il rischio di spezzarsi, se non si fosse fondata su «accordi amichevoli» analoghi a quelli inventati dalla Svizzera.

Di conseguenza, l'annoso e formalistico dibattito relativo all'ambito di applicazione del principio di maggioranza nel Consiglio europeo appare di fatto destituito di un'effettiva rilevanza; per lo stesso motivo, anche il dibattito tra sovranazionalità $\mathrm{e}$ intergovernatività appare sostanzialmente vacuo. Nell'Unione europea, come in Svizzera, la maggioranza può prevalere soltanto su questioni di importanza limitata, quelle in cui, per utilizzare un'espressione ricorrente in questi casi, gli «interessi fondamentali» degli stati-membri non sono in gioco.

Il concetto di regola di maggioranza, quindi, non può giocare un ruolo significativo né nell'Unione europea né in Svizzera. In entrambi i casi, vi è un'ampia varietà di gruppi le cui opinioni devono essere rispettate e conciliate. Facendo un passaggio ulteriore, si potrebbe osservare che l'identità dell'Unione europea può essere costruita soltanto sulla base del riconoscimento della diversità. Come l'identità della Svizzera si è basata sull'orgoglio che i cittadini elvetici attinsero dal fatto di aver raggiunto l'unità in un quadro di (e malgrado la loro) diversità, così l'identità europea potrà svilupparsi attorno al riconoscimento che le diversità, lungi dal configurare un ostacolo, costituiscono il tratto che conferisce all'Unione la sua originalità e quindi la sua forza.

$\mathrm{Da}$ queste premesse discendono numerose conseguenze. 
Anzitutto, si dovrebbe rinunciare al tentativo di introdurre nell'Unione europea dispositivi che prevedano un approccio «maggioritario» anziché consensuale. E quindi opportuno che i Commissari europei siano scelti attraverso un accordo tra gli stati-membri o, ancor meglio, dopo che un accordo congiunto sia stato raggiunto nel Consiglio e nel Parlamento europeo. $\mathrm{La}$ scelta dei commissari non dovrebbe avvenire attraverso elezioni competitive. Essi dovrebbero continuare ad essere nominati per un periodo di tempo definito e la procedura del «voto di fiducia» dovrebbe essere sostituita dal voto consensuale, mentre la procedura del «voto di censura» dovrebbe restare inusitata. Ciò significa che l'attuale prassi di reclutare i commissari dalle principali «famiglie» politiche dell'Unione dovrebbe restare inalterata e, se possibile, essere rafforzata.

La Commissione, tuttavia, dovrebbe essere riformata in due punti. Il numero dei Commissari dovrebbe essere ridotto. Tale riforma potrebbe essere introdotta stabilendo che i commissari siano nominati da «regioni» anziché da singoli paesi, ricomprendendo in ciascuna regione due o più paesi geograficamente contigui e culturalmente affini. L'altra riforma dovrebbe ridurre la durata del mandato del Presidente. Una presidenza di lunga durata può trovare giustificazione sul terreno dell'efficienza, soprattutto fino a quando i poteri della Commissione restassero minacciati. Tale sistema non può però essere mantenuto in un'Unione di quindici, venti o più paesi, poiché la probabilità di ciascun paese di detenere la presidenza diventa estremamente bassa. Il mandato di presidente, pertanto, dovrebbe essere abbreviato e la carica ruotare tra i commissari. Poiché, per le sue dimensioni, non ci si può aspettare che la Commissione sia un organo pienamente collegiale, alla sua guida potrebbe essere insediata informalmente una «troika».

Quanto al Consiglio, esso potrebbe trasformarsi col tempo vieppiù in una seconda camera. Il numero di questioni che tale organismo si troverà ad affrontare diventerà tale che, probabilmente, il suo ruolo tenderà inevitabilmente a ratificare o rigettare proposte anziché a farsene iniziatore. La sola riforma che potrà rendersi necessaria, col risultato di accrescere sensibilmente la trasparenza, è la pubblicazione dei dibattiti.

Non sembra invece esserci un grande bisogno di estendere $i$ poteri del Parlamento europeo. Va da sé che un incremento dei poteri formali di un'istituzione ne accresce lo status e il prestigio e che, da questo punto di vista, i poteri del Parlamento eu- 
ropeo dovrebbero essere in parte estesi. Credere che il Parlamento europeo potrebbe effettivamente esercitare tali poteri, d'altro canto, è pura illusione, in quanto il processo decisionale avrebbe comunque luogo - analogamente a quanto accade in Svizzera - per il tramite di gruppi «consultivi» e di commissioni costituite attorno al Parlamento, al Consiglio e alla Commissione. Così richiede la logica di un sistema consociativo.

$\mathrm{Da}$ ciò si ricavano due inevitabili conseguenze. In primo luogo, come avviene in Svizzera, il processo politico dell'Unione europea sarà piuttosto lento. Nei sistemi parlamentari maggioritari, probabilmente, esso è più rapido, ma poiché l'Unione europea, se vuole evitare di dissolversi, non può essere un sistema parlamentare maggioritario, la lentezza è inevitabile. Perfino nei parlamenti maggioritari, inoltre, il processo politico può essere assai lento quando sono in gioco gli «interessi fondamentali» di qualche settore di popolazione, si tratti del Quebec o dell'Irlanda del Nord. Se la lentezza è il prezzo da pagare per ottenere «accordi amichevoli» e limitare le tensioni all'interno dell'Unione, non si tratta poi di un prezzo troppo elevato.

La seconda conseguenza è che il «deficit democratico» che colpisce il Parlamento europeo non sembra destinato ad essere riassorbito in maniera apprezzabile, in quanto l'assenza di scelte nette al momento delle elezioni le rende poco significative. Si è osservato che molti elettori sono ignari di tale «deficit». Se però fosse chiaro quanto poco le elezioni consentono di influenzare le scelte dell'Unione europea, la loro consapevolezza potrebbe aumentare. Conseguenze analoghe a quelle prodotte da un netto risultato elettorale possono d'altro canto essere ottenute, con gradualità e a spizzico, attraverso la pressione dei gruppi di interesse e delle libere associazioni: la partecipazione all'interno di questi gruppi e associazioni può quindi contribuire a ridurre il «deficit democratico».

Una politica linguistica meno deflagrante. Se nessuna riforma di rilievo degli assetti decisionali dell'Unione europea sembra essere necessaria, la politica linguistica deve essere invece ripensata, seguendo il modello elvetico. In Svizzera, né al ladino, né ai «dialetti» svizzerotedeschi è attribuito lo status delle tre lingue principali e, in molti casi, neppure l'italiano è utilizzato sistematicamente come il tedesco e il francese. Una politica analoga applicata all'Unione europea consiglierebbe di identificare tre livelli distinti: le lingue il cui impiego è continuo e sistematico; le lingue il cui impiego non è sistematico; le lingue ricono- 
sciute, utilizzate nella vita-di-tutti-i-giorni e nella maggior parte dei documenti ufficiali. Ciò renderebbe possibile ridurre a sei un numero ancora elevato ma gestibile - il numero delle lingue «di lavoro» (inglese, francese, tedesco, italiano, spagnolo e olandese), a cui, se la Polonia dovesse far ingresso nell'Unione, andrebbe aggiunto, con identico status, il polacco.

Politica estera $e$ di difesa. L'esperienza svizzera potrebbe tornare assai utile anche sul terreno della politica estera e di difesa. La situazione dei due contesti non potrebbe essere all'apparenza più diversa: la Svizzera è neutrale; molti stati-membri dell'Unione europea non lo sono, sebbene alcuni (Irlanda, Austria, Finlandia) lo siano o tradizionalmente lo siano stati (Svezia). L'Unione europea, inoltre, sperimenta di continuo una profonda divisione, tra gli stati-membri che non sono neutrali, in merito alle politiche da seguire in zone di conflitto quali il Medio Oriente oppure in aree, come l'Africa, in cui uno di essi era stato tradizionalmente dominante. Nel prevedibile futuro, la probabilità che l'Unione europea sia in grado di superare tali divisioni e di adottare una politica estera davvero comune e attiva appare dunque esigua, se non nulla.

In questo quadro, le opzioni di massima si riducono a due. La prima possibilità consiste nell'abbandonare qualsiasi tentativo di definire una politica estera comune, malgrado quanto statuito dal Trattato di Maastricht. Un'ipotesi di questo genere non sembra essere nell'interesse di nessuno, poiché, come si è detto, gli stati-membri non sono in grado di esercitare singolarmente alcuna influenza che oltrepassi i confini della stessa Unione europea. Finché i paesi extracomunitari restano convinti che la politica estera dell'Unione europea è puramente retorica, gli sforzi dei singoli orientati, ad esempio, a esercitare pressioni in aree turbolente saranno destinati a produrre scarsi risultati.

La seconda possibilità è fondata invece sul riconoscimento delle distanze che separano le posizioni degli stati-membri rispetto a una serie di questioni chiave di politica estera, e dell'impossibilità di ricomporle. Ciò suggerisce che l'Unione europea adotti un basso profilo e si concentri su ciò che i suoi membri condividono, vale a dire la sicurezza dell'Unione contro i suoi possibili avversari. Si deve riconoscere che difficilmente gli stati-membri adotterebbero a cuor leggero una linea «minimalista» di questo tipo. Di fatto, però, essi si comportano, e si sono già comportati, come se fossero consapevoli che quella ap- 
pena descritta sia la sola modalità d'azione disponibile. Una linea del genere non è assimilabile al neutralismo elvetico, ma presenta con esso alcuni tratti comuni. La neutralità fu imposta alla Svizzera dal Congresso di Vienna, ma venne accolta dagli svizzeri come una soluzione accettabile, date le divisioni interne e la posizione geografica del paese. Dal punto di vista dell'Unione europea, l'adozione di una politica estera «minimalista» costituirebbe una soluzione altrettanto realistica, tenuto conto delle divisioni interne all'Unione e di una politica mondiale la cui struttura vede ormai un numero non trascurabile di paesi più potenti e popolosi di qualsiasi singolo stato-membro dell'Unione europea, e in grado quindi di esercitare un'influenza non trascurabile nello sviluppo degli affari internazionali.

Il raggio d'azione di questa politica estera «minimalista», inoltre, potrebbe essere progressivamente esteso. Una delle questioni chiave che gli stati-membri devono congiuntamente affrontare è la graduale espansione dell'Unione europea verso Est. L'allargamento pone problemi interni (economici, sociali, nonché politici) ed esterni, poiché chiama in causa i limiti di tale estensione e, in particolare, le relazioni dell'Unione europea nei confronti della Russia. La questione, con tutta evidenza, richiede da parte degli stati-membri la definizione di una posizione comune; tale posizione sarà probabilmente simile alla neutralità svizzera, in quanto per l'Unione europea sarà decisivo evitare di provocare l'ostilità della Russia sostenendo domande «irredentiste».

Nel quadro di una linea «minimalista» inoltre - anche in questo caso sull'esempio svizzero - l'Unione europea potrà far proprio un approccio di politica estera attento ai diritti umani e orientato agli interventi umanitari. Non pochi paesi europei devono il loro scarso prestigio internazionale ad interventi passati non proprio ineccepibili; se l'Unione europea rendesse evidente non solo che quella pagina è stata definitivamente chiusa, ma che la nuova Europa è impegnata sul fronte del rispetto dei diritti umani e dell'erogazione di aiuti intesi a promuovere prosperità e collaborazione su scala mondiale, l'Unione vedrebbe migliorare sensibilmente la propria immagine e potrebbe riconquistare per gradi, questa volta su basi amichevoli, parte dell'influenza che alcuni dei suoi stati-membri detenevano individualmente in passato. Questo approccio «minimalista» alla politica estera presenta insomma per alcuni stati-membri vantaggi evidenti, che tuttavia la tradizione di alcuni ministeri degli esteri e 
la persistente diffidenza di molti paesi extraeuropei nei confronti delle intenzioni dell'Europa rendono difficilmente conseguibili.

Referendum. L'Unione europea ha necessità di accrescere la propria legittimità. Non può fare affidamento soltanto sul sostegno passivo accordato dalla maggior parte dei cittadini, anche se le conseguenze di un sostegno di questo tipo - in assenza di gravi crisi interne o esterne - non sembrano essere dannose. Come si è detto, gli assetti politici dell'Unione europea non dovrebbero essere modificati, poiché il consociativismo deve prevalere. Se tuttavia si vorrà immettere una più diretta partecipazione a livello europeo, il solo modo per farlo è introdurre, sul modello elvetico, dei referendum su scala europea. Pur avendo probabilmente contribuito al declino della partecipazione elettorale, i referendum hanno tuttavia fornito ai cittadini svizzeri un'opportunità di partecipazione di cui vanno orgogliosi. Considerando la condizione dei partiti dell'Unione europea, inoltre, i referendum non produrrebbero l'impatto negativo sull'unità dei partiti che di solito viene loro attribuito. In quasi tutti i paesi dell'Europa occidentale, negli ultimi decenni, l'incidenza dei referendum è in aumento (Paesi Bassi, Lussemburgo, Germania sono i soli paesi tra $\mathrm{i}$ quindici in cui non si è mai svolto alcun referendum su scala nazionale). I referendum su questioni europee, inoltre, hanno suscitato notevole interesse anche se, e probabilmente perché, hanno reso l'Europa una fonte di controversia. Essendo consacrati a questioni specifiche, i referendum su scala europea determinerebbero probabilmente più una divisione nei singoli paesi che non una divisione tra paesi e ciò potrebbe favorire il formarsi di alleanze su scala europea. Coloro che in un caso si trovassero a perdere, infine, possono confidare nel successo in un caso successivo. Questioni relative alle droghe, all'ordine pubblico e all'immigrazione, così come un certo numero di questioni di «coscienza», potrebbero fornire, almeno in una fase inziale, materia sufficiente per questi referendum su scala europea. In presenza di procedure adeguatamente aperte (come il caso dell'Italia può dimostrare), anche referendum su questioni di carattere sociale ed economico - che la discussione attraverso i gruppi e nei parlamenti consente probabilmente di processare in maniera più adeguata - sarebbero quasi certamente accompagnati da un attivo «partecipazionismo». 


\section{Osservazioni conclusive}

La Svizzera può dunque fornire utili indicazioni all'Unione europea e dimostrare, agli scettici come agli entusiasti, che nel cammino verso l'unità le diversità culturali e sociali dell'Europa possono e devono essere preservate. Poiché l'Unione europea si trova essenzialmente a dover definire una via intermedia tra la libertà delle singole componenti di ciò che un tempo era definito il «concerto delle nazioni» - con tutte le sue traumatiche conseguenze - e una centralizzazione, di fatto impossibile, basata su una rigida forma di federalismo, il modello «confederale» elvetico fornisce una soluzione che all'interno dell'Unione europea potrebbe risultare accettabile se non a tutti per lo meno alla grande maggioranza: non obbliga nessuno e consente agli stati-membri di restare artefici del proprio destino e di diventare parziali artefici del destino dell'Europa.

Ciò che dovrebbe rendere pienamente accettabile tale scenario è soprattutto il riconoscimento che il progresso verso l'unità deve essere lento, anche molto lento. L'esperienza della Svizzera mostra che l'unità può essere conseguita soltanto nel lungo periodo. L'Unione europea è iniziata alla metà del ventesimo secolo: suggerire che si dovrà arrivare alla metà del secolo XXI prima di pensare a una vera «unione» non significa certo abusare della pazienza di qualcuno. Basti pensare che alla Svizzera furono necessari almeno cent'anni per passare dall'assetto confederale del 1815 all'unione federale e che per trasformare gli Stati Uniti, che erano una confederazione non ben definita, in una nazione unita ci volle quasi altrettanto (oltreché una crudele guerra civile).

[Traduzione di Aldo Di Virgilio]

\section{Riferimenti bibliografici}

Allemann, F.R. (1985), Vingt-six fois la Suisse, Lausanne, Edition de l'Aire.

Aubert, J.F. (1979), Exposé des institutions politiques de la Suisse à partir de quelques affaires controversées, Lausanne, Payot.

Eschet-Schwarz, A. (1989), The Role of Semi-direct Democracy in Shaping Swiss Federation, in «The Journal of Federalism», XIX, pp. 79-106. 
Knapp, B. (1987), Conféderation et cantons: l'influence des crises sur l'évolution de leurs relations, in «Pouvoirs», 43, pp. 31-47.

Linder, W. (1994), Swiss Democracy, London, Macmillan.

Rokkan, S. (1974), Prefazione a Steiner (1974).

Reif, K. (1985), Ten Second-order National Elections, in K. Reif (a cura di), Ten European Elections, London, Gower.

Sidjansky, D. (1979), Turnout, Stability and the Left-right Dimension, in H.R. Penniman (a cura di), Switzerland at the Polls, Washington D.C., Enterprise Institute, pp. 104-136.

- (a cura di) (1996), L'Union europeénne à l'epreuve du fédéralisme suisse, Genéve.

Siegfried, A. (1947), La Suisse, démocratie témoin, Neuchatel, La Baconnière ( $\mathrm{I}^{\mathrm{a}}$ ed. 1969).

Steiner, J. (1974), Amicable Agreement versus Majority Rule, Chapel Hill, University of North Caroline Press.

Wheare, K.C. (1966), Federalism, Oxford, Oxford University Press. 